Canadian University Music Review

Revue de musique des universités canadiennes

Dominique Jameux. Pierre Boulez. Paris : Fayard/Sacem, 1984, 492 pp., nombreux exemples musicaux, 18 photographies

\title{
Jean-Jacques Nattiez
}

Numéro 6, 1985

URI : https://id.erudit.org/iderudit/1014067ar

DOI : https://doi.org/10.7202/1014067ar

Aller au sommaire du numéro

Éditeur(s)

Canadian University Music Society / Société de musique des universités

canadiennes

ISSN

0710-0353 (imprimé)

2291-2436 (numérique)

Découvrir la revue

Citer ce compte rendu

Nattiez, J.-J. (1985). Compte rendu de [Dominique Jameux. Pierre Boulez. Paris :

Fayard/Sacem, 1984, 492 pp., nombreux exemples musicaux, 18

photographies]. Canadian University Music Review / Revue de musique des

universités canadiennes, (6), 319-321. https://doi.org/10.7202/1014067ar

(C) Canadian University Music Society / Société de musique des universités canadiennes, 1985
Ce document est protégé par la loi sur le droit d'auteur. L'utilisation des services d'Érudit (y compris la reproduction) est assujettie à sa politique d'utilisation que vous pouvez consulter en ligne.

https://apropos.erudit.org/fr/usagers/politique-dutilisation/ 


\section{COMPTES RENDUS/REVIEWS}

DominiQue Jameux. Pierre Boulez. Paris : Fayard/Sacem, 1984, 492 pp., nombreux exemples musicaux, 18 photographies.

A coup sûr, voici un livre essentiel, par la qualité de la recherche, de la documentation, du style ; par l'importance du sujet aussi. Car au fil des années, Pierre Boulez s'est imposé non seulement comme un des compositeurs les plus marquants du XXe siècle - peut-être le plus décisif des trente dernières années, vu la place que sa dernière oeuvre, Répons, s'apprête à occuper dans l'histoire de la musique -, mais aussi comme figure dominante de la vie musicale française et internationale : comme chef-d'orchestre, pédagogue, conférencier, essayiste et animateur de diverses institutions. Boulez méritait sans doute plus que le petit ouvrage de Paul Griffiths et surtout mieux que le brulot pseudo-psychanalytique de Joan Peyser. Dominique Jameux, déjà connu pour ses livres sur Strauss et Berg, ses nombreux articles et préfaces et, en France, pour son rôle d'animateur apprécié à Radio-France, nous apporte le premier ouvrage de fond qui couvre les diverses facettes de la carrière de Boulez. Le livre se divise en deux grandes parties. La première est consacrée à la " trajectoire " biographique sous tous ses aspects. La seconde présente une série de douze (nombre involontaire?) analyses d'oeuvres sélectionnées.

L'auteur ne se défend pas du caractère journalistique de la première partie, mais en admettant que ce soit une tare, on le lui pardonne d'autant mieux qu'il le fait avec verve et talent. Visiblement, Jameux aime son modèle ( "le seul musicien [actuel] qui mérite une telle dépense d'énergie"), mais l'admiration ne le rend jamais aveugle : outre des " notes pour un portrait " qui traitent avec élégance de la personnalité souvent controversée de Boulez et qui ne ressemblent en rien à une hagiographie, l'auteur ne cache pas ses réserves quand il le croit nécessaire : sensibilité de Boulez pour certains hochets officiels, erreurs diplomatiques, absorption dans des tâches administratives stérilisantes. C'est que, pour Jameux, l'essentiel de Boulez réside dans ses oeuvres et son activité musicales. Nous tenons enfin un livre où l'on trouvera, avec précision et un grand bonheur dans le choix des formules, la chronologie de la carrière boulezienne et la description des moments marquants de son activité créatrice et artistique. Toutes les oeuvres sont au moins évoquées dans la première partie, donc resituées dans le contexte qui les a vu naître - pensée de Boulez et vie musicale du temps, avec souvent un florilège des critiques les plus significatives qui les accueillirent. Pas de technique, ni d'exemples musicaux dans cette première partie: les analyses sont regroupées dans la seconde. La section documentaire de l'ouvrage suffirait 
à en faire un livre de référence indispensable : elle comprend un catalogue des oeuvres, avec la date de création, le lieu d'édition, et surtout la chronologie des diverses versions des nombreuses "Works in progress" qui se sont étalées dans le temps ; une discographie complète de Boulez compositeur et chef d'orchestre ; une filmographie, et une bibliographie -à laquelle il manque peut-être une liste complète des écrits.

Ouvrage décisif, le Boulez de Jameux n'est sans doute pas définitif. On sent que l'auteur n'a pas eu le temps d'investigations prolongées sur place à Darmstadt, à Londres, à Cleveland et à New York. Parce que, devant la stature de Boulez, on aurait presque envie d'exiger de Jameux qu'il soit un Glasenapp ou un Spitta, on regrette parfois de ne pas en savoir plus - après une plongée en archives ou des entrevues systématiques de témoins - sur les programmes du chef d'orchestre avec les grandes formations londoniennes et américaines, sur le contenu des cours de Darmstadt et de Bâle (de quoi parlait-il, au juste?) ou du Collège de France (dont il existe au moins les résumés dans l'annuaire). Par là, le livre ressortit davantage à la musicographie qu'à la musicologie. Mais notre réserve la plus forte, de ce point de vue, porterait sur la description de la trajectoire musicale proprement dite. Car si Jameux décrit avec efficacité l'ascension de Boulez, en revanche, on sent moins bien comment, au niveau de la pensée, Boulez part de la dissociation des paramètres, c'est-à-dire de la discrépance entre les innovations de chacun de ses prédécesseurs et les aspects plus archaïsants de leur langage, pour rechercher, quasi-désespérément parfois, la fusion du matériau et de l'invention, pour déboucher sur Répons, produit à la fois d'une institution, d'une technologie et d'un langage. Cette convergence, on aimerait presque dire cette nécessité au sens des allemands (Bedürfnis) qui représente pour nous la trame fondamentale de la longue marche boulezienne, Jameux en donne certes les éléments (notamment p. 77, p. 107, p. 204), mais la construction du livre ne nous semble pas mettre en évidence la trajectoire irrésistible qui aboutit à Répons. Du reste, l'hypothèse selon laquelle cette dernière oeuvre serait reliée à la crise interne de l'IRCAM en 1980 (p. 254, p. 261) nous paraît fort discutable. Dernière réserve, à propos d'un livre qui n'en mérite pas tant: nulle part Jameux n'analyse le lien entre l'attitude devant les oeuvres dirigées et l'esthétique de Boulez compositeur, alors que deux cents cinquante pages des Points de repère, le dernier ouvrage du compositeur, sont parfaitement explicites à ce sujet.

La seconde partie rassemble douze analy ses d'oeuvres et représente, selon nous, un petit tour de force. Car, on le sait, la plupart des oeuvres de Boulez sont trop complexes pour permettre une analyse de détail. Jameux - on reconnait ici l'homme de radio - a donc choisi le point de vue de l'auditeur : chaque pièce fait l'objet d'une sorte de fiche technique, avec la présentation de son déroulement, des séries de base quand elles sont accessibles. On trouve aussi des plans chronométrés des oeuvres selon leurs grandes sections. Le souci pédagogique est évident et, sur un autre plan, il témoigne des mêmes qualités de communicateur dont Jameux fait 
preuve dans la première partie. C'est dire, au total, qu'à propos d'un compositeur réputé difficile, l'auteur a su trouver aux embûches successives les solutions élégantes. En cela, il a écrit un des ouvrages les plus importants qu'on puisse lire actuellement sur la musique contemporaine.

Jean-Jacques Nattiez

Revue internationale de musique française, 4e année, février $1983, \mathrm{~N}^{\circ} 10$.

L'information musicale, considérée du point de vue heuristique, ne se trouve pas forcément là où, d'emblée, on s'imaginerait la trouver. Telle pourrait être, grosso modo, l'idée-maîtresse qui a conduit Danièle Pistone dans un copieux article de cette livraison (pp. 7-54), à s'atteler à l'étude du Charivari, quotidien satirique français (d'un format d'environ quatre pages) qui naquit le $1^{\mathrm{er}}$ décembre 1832 puis disparut, déjà centenaire, en 1937.

Concentrant sa recherche historiographique sur la période comprise entre 1832 et 1870 - tout en souhaitant par ailleurs que d'autres chercheurs complètent l'entreprise pour les années subséquentes -, Danièle Pistone a voulu tâter le pouls de la vie musicale française au siècle dernier et ce, à travers le prisme d'un journal en marge de la presse musicale spécialisée de l'époque.

De fait, si la Revue musicale, le Journal des Débats ou encore le Corsaire et l'Entracte rendent déjà compte des événements musicaux du temps, le Charivari pour sa part entend aborder l'art sonore dans une optique résolument anti-conformiste, fidèle en cela à sa pensée politique contestataire ainsi qu'à sa prédilection pour la caricature sociale.

Parce que le monde musical fait lui aussi partie intégrante de la société, il ne pouvait donc échapper à l'esprit caustique du Charivari. D'autant plus que la qualité et la nature des informations sur la vie musicale consignées par ce journal furent à ce point significatives que celles-ci "méritaient", selon Pistone, de faire l'objet d'un dossier spécifique.

Danièle Pistone a choisi de découper son article à partir des trois rubriques suivantes : 1) " institutions et leur public ";2) " des musiciens aux instruments "; 3) " les goûts et les genres ". Au surplus, des croquis lithographiés de Cham et Daumier (parus alors dans le Charivari) ont été incorporés au dossier; heureuse initiative car ils parviennent à bien exprimer, en raccourci, toute la saveur originale du journal.

Dans la première partie consacrée aux institutions et leur public, Pistone s'emploie à distinguer les types d'établissements (au sens large) dont traîte le Charivari. On apprend alors que le Conservatoire, l'Opéra, l'Opéra-comique et une diversité d'autres salles possèdent bien d'autres at traits (outre l'art en lui-même) pour capter l'at tention des chroniqueurs 